\title{
Landesman-Lazer type condition for second-order differential equations at resonance with impulsive effects
}

Jin $\mathrm{Li}^{1 *}$ and Meilin Zheng ${ }^{2}$

${ }^{*}$ Correspondence:

lijin7912@gmail.com

'School of Science, Jiujiang

University, Jiujiang, 332005, China

Full list of author information is

available at the end of the article

\section{Abstract}

In this paper, we study the existence of periodrc solutions of second-order impulsive differential equations at resonance. We prove the existeace of periodic solutions under a generalized Landesman-Lazer type condition by using the variational method. The impulses can generate a periodic solution.

Keywords: impulsive differential equations; Landesman-Lazer type condition; variational method

\section{Introduction}

We are concerned with periodic boundary value problem of second-order impulsive differential equations at resonance

$$
\left\{\begin{array}{l}
x^{\prime \prime}(t)+m^{2} x(t)+f(t, x(t))=e(t), \quad \text { a.e. } t \in[0,2 \pi], \\
x(0)-x(2 \pi)=x^{\prime}(0)-x^{\prime}(2 \pi)=0, \\
x\left(t_{j}^{+}\right)=x\left(t_{j}^{-}\right), \\
\Delta x^{\prime}\left(t_{j}\right):=x^{\prime}\left(t_{j}^{+}\right)-x^{\prime}\left(t_{j}^{-}\right)=I_{j}\left(t_{j}, x\left(t_{j}\right)\right), \quad j=1,2, \ldots, p,
\end{array}\right.
$$

where $m \in \mathbb{N}, f:[0,2 \pi] \times \mathbb{R} \rightarrow \mathbb{R}$ is a Carathéodory function, $e \in L^{1}(0,2 \pi), 0<t_{1}<t_{2}<$ $\cdots<t_{p}<2 \pi$, and $I_{j}:[0,2 \pi] \times \mathbb{R} \rightarrow \mathbb{R}$ is continuous for every $j$.

When $\Delta x^{\prime}\left(t_{j}\right) \equiv 0$, problem (1.1) becomes to the well-known periodic boundary value problem at resonance

$$
\left\{\begin{array}{l}
x^{\prime \prime}(t)+m^{2} x(t)+f(t, x(t))=e(t), \quad \text { a.e. } t \in[0,2 \pi] \\
x(0)-x(2 \pi)=x^{\prime}(0)-x^{\prime}(2 \pi)=0
\end{array}\right.
$$

There are many existence results for problem (1.2) in the literature. Let us mention some pioneering works by Lazer [1], Lazer and Leach [2], and Landesman and Lazer [3]. In [3], a key sufficient condition for the existence of solutions of problem (1.2) is the so-called 
Landesman-Lazer condition,

$$
\begin{aligned}
\int_{0}^{2 \pi} e(t) \sin (m t+\theta) d t< & \int_{0}^{2 \pi}\left[\left(\liminf _{x \rightarrow+\infty} f(t, x)\right) \sin ^{+}(m t+\theta)\right. \\
& \left.-\left(\limsup _{x \rightarrow-\infty} f(t, x)\right) \sin ^{-}(m t+\theta)\right] d t, \quad \forall \theta \in \mathbb{R}
\end{aligned}
$$

where $\sin ^{ \pm}(m t+\theta)=\max \{ \pm \sin (m t+\theta), 0\}$.

It is well known that the theory of impulsive differential equations has been recognized) to not only be richer than that of differential equations without impulses, but also to provide a more adequate mathematical model for numerous processes and phenomenastudied in physics, biology, engineering, etc. We refer the reader to the book [4]. Recently, the Dirichlet and periodic boundary conditions problems for second-order differential equations with impulses in the derivative and without impulses are studied by some authors via variational method [5-11]. In this paper, we will investigate problem (1.1) under a more general Landesman-Lazer type condition. Define

$$
F(t, x)=\int_{0}^{x} f(t, s) d s, \quad F_{+}(t)=\liminf _{x \rightarrow+\infty} \frac{F(t, x)}{x}, \quad F(t)=\limsup _{x \rightarrow-\infty} \frac{F(t, x)}{x}
$$

and for $j=1,2, \ldots, p$,

$$
J_{j}(t, x)=\int_{0}^{x} I_{j}(t, s) d s, \quad \limsup _{x \rightarrow+\infty} \frac{f^{\prime}(t, x)}{x}, \quad J_{j}^{-}(t)=\liminf _{x \rightarrow-\infty} \frac{J_{j}(t, x)}{x} .
$$

Throughout this paper, we give the following fundamental assumptions.

$\left(\mathrm{H}_{1}\right)$ There exists $p \in L^{1}([0,2 \pi],[0,+\infty))$ such that $|f(t, x)| \leq p(t)$, for a.e. $t \in[0,2 \pi]$ and for all $x \in \mathbb{R}$.

$\left(\mathrm{H}_{2}\right)$ There exist positive constants $c_{1}, c_{2}, \ldots, c_{p}$ such that for all $t, x \in \mathbb{R}$,

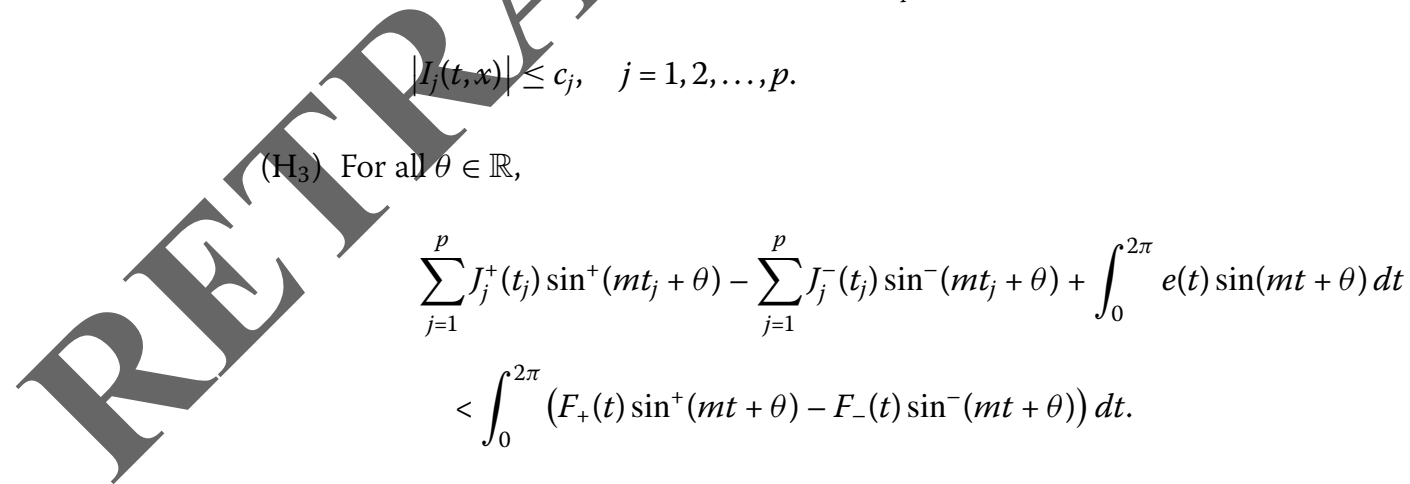

We now can state the main theorem of this paper.

Theorem 1.1 Assume that the conditions $\left(\mathrm{H}_{1}\right),\left(\mathrm{H}_{2}\right)$, and $\left(\mathrm{H}_{3}\right)$ hold. Then problem (1.1) has at least one $2 \pi$-periodic solution.

To demonstrate the impulsive effects clearly, we can take

$$
I_{j}(t, x) \equiv d_{j}, \quad j=1,2, \ldots, p
$$

where $d_{1}, d_{2}, \ldots, d_{p}$ are constants. Hence, $J_{j}^{ \pm}(t)=d_{j}$. 
From Theorem 1.1, we obtain the following result.

Corollary 1.2 Assume that we have the conditions $\left(\mathrm{H}_{1}\right),(1.4)$, and the following.

$\left(\mathrm{H}_{3}^{\prime}\right)$ For all $\theta \in \mathbb{R}$

$$
\begin{aligned}
& \sum_{j=1}^{p} d_{j} \sin \left(m t_{j}+\theta\right)+\int_{0}^{2 \pi} e(t) \sin (m t+\theta) d t \\
& \quad<\int_{0}^{2 \pi}\left(F_{+}(t) \sin ^{+}(m t+\theta)-F_{-}(t) \sin ^{-}(m t+\theta)\right) d t
\end{aligned}
$$

hold. Then problem (1.1) has at least one $2 \pi$-periodic solution.

Moreover, we have the following corollary.

Corollary 1.3 Assume that we have the conditions $\left(\mathrm{H}_{1}\right)$ ald the following.

$\left(\mathrm{H}_{3}^{\prime \prime}\right)$ For all $\theta \in \mathbb{R}$,

$$
\left.\int_{0}^{2 \pi} e(t) \sin (m t+\theta) d t \int_{0}^{2 \pi}(t) \sin ^{4}(m t+\theta)-F_{-}(t) \sin ^{-}(m t+\theta)\right) d t
$$

holds. Then problem (1.2) has at least gne $2 \pi$-periodic solution.

Remark 1.4 By a simple calculation, one can easily derive

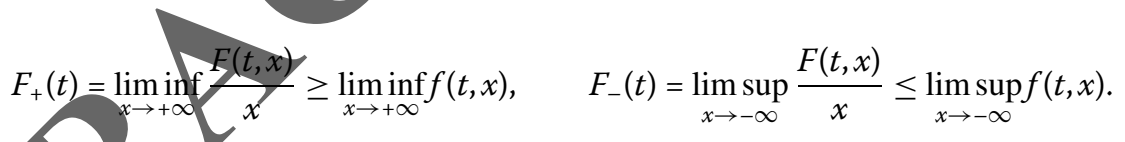

A simple example $f(t, x)=\sin t+\cos x$ illustrates it. Thus condition $\left(\mathrm{H}_{3}^{\prime \prime}\right)$ generalizes condition (1.3). Hence, our results improve the related results in the literature mentioned above. Moreoyer, since we consider the problem with impulses, Theorem 1.1 is also a complement of the pioneering works.

Remark 1.5 It is remarkable that Landesman-Lazer condition $\left(\mathrm{H}_{3}^{\prime \prime}\right)$ is an 'almost' necessary and sufficient condition when $F_{+}$and $F_{-}$are replaced by $f_{+}$and $f_{-}$, where $f_{+}=$ $\lim _{x \rightarrow+\infty} f(t, x), f_{-}=\lim _{x \rightarrow-\infty} f(t, x)$, and $f_{-}(t) \leq f(t, x) \leq f_{+}(t)$ (see [12, p.70]). If the condition (1.5) is not satisfied, i.e., $\exists \theta \in \mathbb{R}$,

$$
\int_{0}^{2 \pi} e(t) \sin (m t+\theta) d t \geq \int_{0}^{2 \pi}\left(F_{+}(t) \sin ^{+}(m t+\theta)-F_{-}(t) \sin ^{-}(m t+\theta)\right) d t
$$

problem (1.2) cannot be guaranteed to have periodic solution. For example, we consider resonant differential equation

$$
x^{\prime \prime}+m^{2} x+(1+\sin m t) \arctan x=8 \sin m t .
$$


Obviously, $f(t, x)=(1+\sin m t) \arctan x, e(t)=8 \sin m t$, and $F_{+}(t)=\frac{\pi}{2}(1+\sin m t), F_{-}(t)=$ $-\frac{\pi}{2}(1+\sin m t)$. Taking $\theta=0$, we have

$$
\begin{aligned}
& \int_{0}^{2 \pi} e(t) \sin m t d t-\int_{0}^{2 \pi}\left(F_{+}(t) \sin ^{+} m t-F_{-}(t) \sin ^{-} m t\right) d t \\
& \quad=8 \pi-\frac{\pi}{2} \int_{0}^{2 \pi}(1+\sin m t)|\sin m t| d t \\
& \quad \geq 8 \pi-2 \pi^{2}>0 .
\end{aligned}
$$

Then $\left(\mathrm{H}_{3}^{\prime \prime}\right)$ is not satisfied. From now on, we prove that $(1.6)$ has not $2 \pi$-periodic solution by contradiction. Assume that (1.6) has $2 \pi$-periodic solution. Multiplying both sides of (1.6) by $\sin m t$ and integrating over $[0,2 \pi]$, we get

$$
\begin{aligned}
8 \pi & =\int_{0}^{2 \pi}(1+\sin m t) \arctan x \sin m t d t \\
& \leq \int_{0}^{2 \pi}|(1+\sin m t) \arctan x \cos m t| d t \\
& \leq \pi \int_{0}^{2 \pi} d t=2 \pi^{2}
\end{aligned}
$$

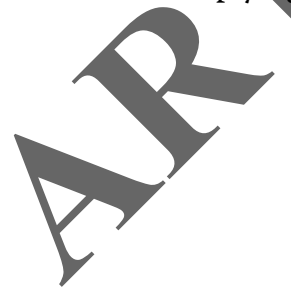

which is impossible. Hence, problem (1.2) may have no solution if the condition $\left(\mathrm{H}_{3}^{\prime \prime}\right)$ is not satisfied. However, as long as $\left(\mathrm{H}_{3}\right)$ holds, problem $(1.1)$ will have at least one periodic solution. Therefore, the impulses can generate a periodic solution.

The rest of the paper is organized as follows. In Section 2, we shall state some notations, some necessary definitions, and a saddle theorem due to Rabinowitz. In Section 3, we shall prove Theorem 1 .

\section{Preliminaries}

In the following, we introduce some notations and some necessary definitions.

Define

$$
H=\left\{x \in H^{1}(0,2 \pi): x(0)=x(2 \pi)\right\}
$$

with the norm

$$
\|x\|=\left(\int_{0}^{2 \pi}\left(x^{\prime 2}(t)+x^{2}(t)\right) d t\right)^{\frac{1}{2}} .
$$

Consider the functional $\varphi(x)$ defined on $H$ by

$$
\begin{aligned}
\varphi(x)= & \frac{1}{2} \int_{0}^{2 \pi} x^{\prime 2}(t) d t-\frac{m^{2}}{2} \int_{0}^{2 \pi} x^{2}(t) d t-\int_{0}^{2 \pi} F(t, x(t)) d t \\
& +\int_{0}^{2 \pi} e(t) x(t) d t+\sum_{j=1}^{p} J_{j}\left(t_{j}, x\left(t_{j}\right)\right) .
\end{aligned}
$$


Similarly as in [7], $\varphi(x)$ is continuously differentiable on $H$, and

$$
\begin{aligned}
\varphi^{\prime}(x) v(t)= & \int_{0}^{2 \pi} x^{\prime}(t) v^{\prime}(t) d t-m^{2} \int_{0}^{2 \pi} x(t) v(t) d t-\int_{0}^{2 \pi} f(t, x(t)) v(t) d t \\
& +\int_{0}^{2 \pi} e(t) v(t) d t+\sum_{j=1}^{p} I_{j}\left(t_{j}, x\left(t_{j}\right)\right) v\left(t_{j}\right), \quad \text { for } \forall v(t) \in H .
\end{aligned}
$$

Now, we have the following lemma.

Lemma 2.1 If $x \in H$ is a critical point of $\varphi$, then $x$ is a $2 \pi$-periodic solution of (1.1).

The proof of Lemma 2.1 is similar to Lemma 2.1 in [6], so we omit it.

We say that $\varphi$ satisfies (PS) if every sequence $\left(x_{n}\right)$ for which $\varphi\left(x_{n}\right)$ is bounded in $\mathbb{R}$ and $\varphi^{\prime}\left(x_{n}\right) \rightarrow 0($ as $n \rightarrow \infty)$ possesses a convergent subsequence.

To prove the main result, we will use the following saddle point theorem due to Rabinowitz [13] (or see [12]).

Theorem 2.2 Let $\varphi \in C^{1}(H, \mathbb{R})$ and $H=H \quad \oplus H^{+}, \operatorname{dim}\left(H^{-}\right)<\infty, \operatorname{dim}\left(H^{+}\right)=\infty$. We suppose that:

(a) There exists a bounded neighborhood Dof 0 in $H^{-}$and a constant $\alpha$ such that

$$
\left.\varphi\right|_{\partial D} \leq \alpha
$$

(b) there exists a constant $\beta>\alpha$ such that $\left.\varphi\right|_{H^{+}} \geq \beta$;

(c) $\varphi$ satisfies (PS).

Then the functional $\varphi$ has a critical point in $H$.

\section{The proof of Theorem 1.1}

In this section, we frest show that the functional $\varphi$ satisfies the Palais-Smale condition.

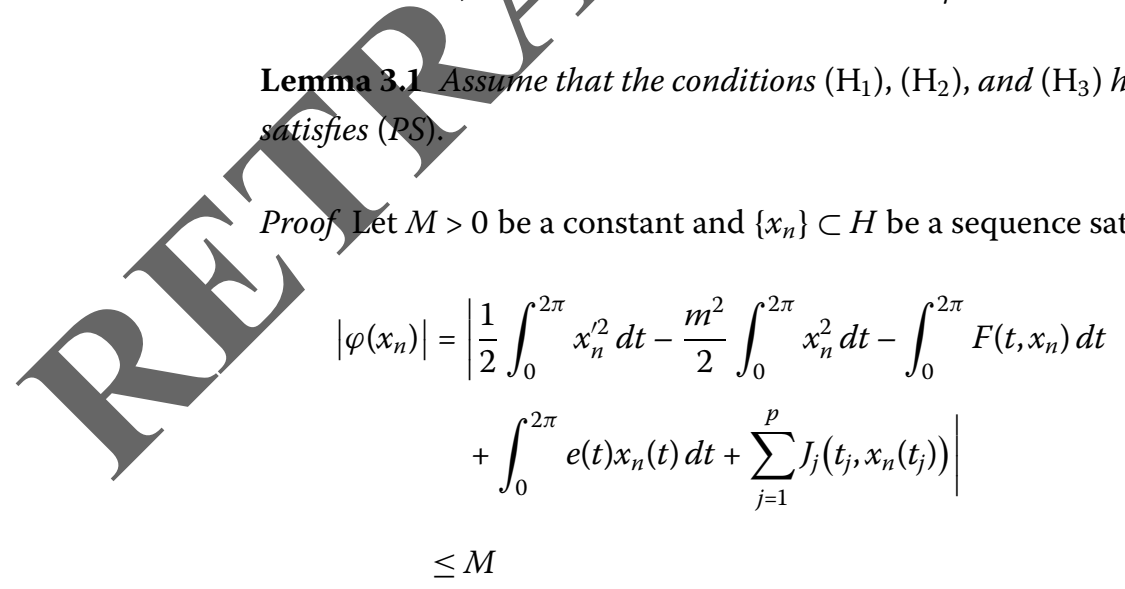

Lemma 3.1 Assume that the conditions $\left(\mathrm{H}_{1}\right),\left(\mathrm{H}_{2}\right)$, and $\left(\mathrm{H}_{3}\right)$ hold. Then $\varphi$ defined by

and

$$
\lim _{n \rightarrow \infty}\left\|\varphi^{\prime}\left(x_{n}\right)\right\|=0 .
$$

We first prove that $\left\{x_{n}\right\}$ is bounded in $H$ by contradiction. Assume that $\left\{x_{n}\right\}$ is unbounded. Let $\left\{z_{k}\right\}$ be an arbitrary sequence bounded in $H$. It follows from (3.2) that, for 
any $k \in \mathbb{N}$,

$$
\lim _{n \rightarrow \infty}\left|\varphi^{\prime}\left(x_{n}\right) z_{k}\right| \leq \lim _{n \rightarrow \infty}\left\|\varphi^{\prime}\left(x_{n}\right)\right\|\left\|z_{k}\right\|=0 .
$$

Thus

$$
\lim _{n \rightarrow \infty} \varphi^{\prime}\left(x_{n}\right) z_{k}=0 \quad \text { uniformly for } k \in \mathbb{N} \text {. }
$$

Hence,

$$
\begin{aligned}
& \lim _{n \rightarrow \infty}\left(\int_{0}^{2 \pi}\left(x_{n}^{\prime} z_{k}^{\prime}-m^{2} x_{n} z_{k}\right) d t-\int_{0}^{2 \pi}\left(f\left(t, x_{n}\right) z_{k}-e(t) z_{k}\right) d t\right. \\
& \left.\quad+\sum_{j=1}^{p} I_{j}\left(t_{j}, x_{n}\left(t_{j}\right)\right) z_{k}\left(t_{j}\right)\right)=0 .
\end{aligned}
$$

By $\left(\mathrm{H}_{1}\right)$ and $\left(\mathrm{H}_{2}\right)$, we have

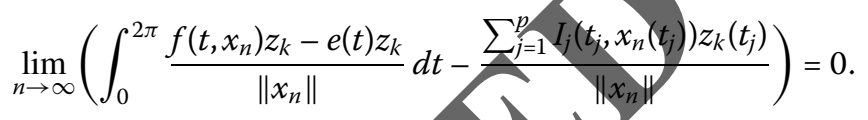

From (3.3) and (3.4), we obtain

$$
\lim _{n \rightarrow \infty} \int_{0}^{2 \pi}\left(\frac{x_{n}^{\prime}}{\left\|x_{n}\right\|} z_{k}^{\prime}-m^{2} \frac{x_{n}}{\left\|x_{n}\right\|} z_{k}\right) d t=0 .
$$

Set

$$
\text { and furthermore, }
$$

Replacing $z_{k}$ in (3.6) by $\left(y_{n}-y_{i}\right)$, we get

$$
\lim _{\substack{n \rightarrow \infty \\ i \rightarrow \infty}}\left(\left\|y_{n}-y_{i}\right\|^{2}-\left(m^{2}+1\right)\left\|y_{n}-y_{i}\right\|_{2}^{2}\right)=0 .
$$

Due to the compact embedding $H \hookrightarrow L^{2}(0,2 \pi)$, going to a subsequence,

$$
y_{n} \rightarrow y_{0} \quad \text { weakly in } H, \quad y_{n} \rightarrow y_{0} \quad \text { in } L^{2}(0,2 \pi) .
$$


Therefore,

$$
\lim _{\substack{n \rightarrow \infty \\ i \rightarrow \infty}}\left\|y_{n}-y_{i}\right\|_{2}^{2}=0
$$

Furthermore, we have

$$
\lim _{\substack{n \rightarrow \infty \\ i \rightarrow \infty}}\left\|y_{n}-y_{i}\right\|^{2}=0
$$

which implies $\left(y_{n}\right)$ is Cauchy sequence in $H$. Thus, $y_{n} \rightarrow y_{0}$ in $H$. It follows from (3.5) and the usual regularity argument for ordinary differential equations (see [14]) that

$$
y_{0}=k_{1} \sin m t+k_{2} \cos m t
$$

where $k_{1}^{2}+k_{2}^{2}=\frac{1}{\left(m^{2}+1\right) \pi}\left(\left\|y_{0}\right\|=1\right)$. (Different subsequences of $\left\{y_{n}\right\}$ eorrespond to different $k_{1}$ and $k_{2}$.)

Write (3.7) as

$$
y_{0}=\frac{1}{\sqrt{\left(m^{2}+1\right) \pi}} \sin (m t+\theta)
$$

where $\theta$ satisfies $\sin \theta=\frac{k_{2}}{\sqrt{k_{1}^{2}+k_{2}^{2}}}$ and $\cos \theta=\frac{k_{1}}{\sqrt{k_{1}^{2}+k_{2}^{2}}}$.

Taking $z_{k}=\frac{1}{\sqrt{\left(m^{2}+1\right) \pi}} \sin (m t+\theta)$, we get, for any $n \in \mathbb{N}$,

$$
\int_{0}^{2 \pi}\left(x_{n}^{\prime} z_{k}^{\prime}-m^{2} x_{n} z_{k}\right) d t=0 \text {. }
$$

Thus, itfollows from (3.3) and (3.8) that

$$
\lim _{n \rightarrow \infty}\left[\int_{0}^{2 \pi}\left(f\left(t, x_{n}\right)-e(t)\right) \frac{1}{\sqrt{\left(m^{2}+1\right) \pi}} \sin (m t+\theta) d t\right.
$$

$$
\left.-\sum_{j=1}^{p} I_{j}\left(t_{j}, x_{n}\left(t_{j}\right)\right) \frac{1}{\sqrt{\left(m^{2}+1\right) \pi}} \sin \left(m t_{j}+\theta\right)\right]=0 .
$$

By $\left(\mathrm{H}_{1}\right)$ and $\left(\mathrm{H}_{2}\right)$, we obtain

$$
\begin{gathered}
\lim _{n \rightarrow \infty}\left[\int_{0}^{2 \pi}\left(f\left(t, x_{n}\right)-e(t)\right)\left(\frac{1}{\sqrt{\left(m^{2}+1\right) \pi}} \sin (m t+\theta)-y_{n}\right) d t\right. \\
\left.-\sum_{j=1}^{p} I_{j}\left(t_{j}, x_{n}\left(t_{j}\right)\right)\left(\frac{1}{\sqrt{\left(m^{2}+1\right) \pi}} \sin \left(m t_{j}+\theta\right)-y_{n}\left(t_{j}\right)\right)\right]=0 .
\end{gathered}
$$

It follows from (3.9) and (3.10) that

$$
\lim _{n \rightarrow \infty}\left[\int_{0}^{2 \pi}\left(f\left(t, x_{n}\right)-e(t)\right) y_{n} d t-\sum_{j=1}^{p} I_{j}\left(t_{j}, x_{n}\left(t_{j}\right)\right) y_{n}\left(t_{j}\right)\right]=0 .
$$


Hence, replacing $z_{k}$ in (3.3) by $y_{n}$, we have

$$
\lim _{n \rightarrow \infty} \int_{0}^{2 \pi}\left(x_{n}^{\prime} \frac{x_{n}^{\prime}}{\left\|x_{n}\right\|}-m^{2} x_{n} \frac{x_{n}}{\left\|x_{n}\right\|}\right) d t=0 .
$$

Now, dividing (3.1) by $\left\|x_{n}\right\|$, we get

$$
\begin{aligned}
\frac{-M}{\left\|x_{n}\right\|} & \leq \frac{1}{2} \int_{0}^{2 \pi}\left(\frac{x_{n}^{\prime 2}}{\left\|x_{n}\right\|}-\frac{m^{2} x_{n}^{2}}{\left\|x_{n}\right\|}\right) d t-\int_{0}^{2 \pi} \frac{F\left(t, x_{n}\right)-e(t) x_{n}}{\left\|x_{n}\right\|}+\frac{\sum_{j=1}^{p} J_{j}\left(t_{j}, x_{n}\left(t_{j}\right)\right)}{\left\|x_{n}\right\|} \\
& \leq \frac{M}{\left\|x_{n}\right\|}
\end{aligned}
$$

which yields

$$
\int_{0}^{2 \pi} \frac{F\left(t, x_{n}\right)-e(t) x_{n}}{\left\|x_{n}\right\|} \leq \frac{M}{\left\|x_{n}\right\|}+\frac{1}{2} \int_{0}^{2 \pi}\left(\frac{x_{n}^{\prime 2}}{\left\|x_{n}\right\|}-\frac{m^{2} x_{n}^{2}}{\left\|x_{n}\right\|}\right) d t+\frac{\sum_{j=1}^{p} J_{j}\left(t_{j}, x_{n}\left(t_{j}\right)\right)}{\left\|x_{n}\right\|} .
$$

Note that $\frac{x_{n}}{\left\|x_{n}\right\|} \rightarrow \frac{1}{\sqrt{\left(m^{2}+1\right) \pi}} \sin (m t+\theta)$ in $H$. Due to the compact embedding $H \hookrightarrow C(0,2 \pi)$ and $\left|x_{n}(t)\right| \rightarrow+\infty$, we have $\frac{x_{n}}{\left\|x_{n}\right\|} \rightarrow \frac{1}{\sqrt{\left(m^{2}+1\right) \pi}} \sin (m t+\theta)$ in $C(0,2 \pi)$. Furthermore,

$$
\lim _{n \rightarrow \infty} x_{n}(t)= \begin{cases}+\infty, & \forall t \in I_{+}:=\{t \in[0,2 \pi] \mid \sin (m t+\theta)>0\}, \\ -\infty, & \forall t \in I_{-}:=\{t \in[0,2 \pi] \mid \sin (m t+\theta)<0\} .\end{cases}
$$

Hence, from (3.11) and (3.12), we have

$$
\begin{array}{r}
\quad \limsup _{n \rightarrow \infty} \sum_{j=1}^{p} \frac{J_{j}\left(t_{j}, x_{n}\left(t_{j}\right)\right)}{x_{n}\left(t_{j}\right)} \cdot \frac{x_{n}^{+}\left(t_{j}\right)}{\left\|x_{n}\right\|} \\
\liminf _{n \rightarrow \infty} \int_{0}^{2 \pi} \frac{F\left(t, x_{n}\right)-e(t) x_{n}}{\left\|x_{n}\right\|} d t \leq \liminf _{n \rightarrow \infty} \sum_{j=1}^{p} \frac{J_{j}\left(t_{j}, x_{n}\left(t_{j}\right)\right)}{x_{n}\left(t_{j}\right)} \cdot \frac{x_{n}^{+}\left(t_{j}\right)-x_{n}^{-}\left(t_{j}\right)}{\| \liminf _{n \rightarrow \infty} \sum_{j=1}^{p} \frac{J_{j}\left(t_{j}, x_{n}\left(t_{j}\right)\right)}{x_{n}\left(t_{j}\right)} \cdot \frac{x_{n}^{-}\left(t_{j}\right)}{\left\|x_{n}\right\|}} \\
=\frac{1}{\sqrt{\left(m^{2}+1\right) \pi}} \sum_{j=1}^{p} J_{j}^{+}\left(t_{j}\right) \sin ^{+}\left(m t_{j}+\theta\right) \\
-\frac{1}{\sqrt{\left(m^{2}+1\right) \pi}} \sum_{j=1}^{p} J_{j}^{-}\left(t_{j}\right) \sin ^{-}\left(m t_{j}+\theta\right) .
\end{array}
$$

Using Fatou's lemma, we get

$$
\begin{aligned}
\liminf _{n \rightarrow \infty} \int_{0}^{2 \pi} \frac{F\left(t, x_{n}\right)}{\left\|x_{n}\right\|} d t & =\liminf _{n \rightarrow \infty}\left[\int_{I_{+}} \frac{F\left(t, x_{n}\right)}{x_{n}} \frac{x_{n}}{\left\|x_{n}\right\|} d t-\int_{I_{-}} \frac{F\left(t, x_{n}\right)}{x_{n}} \frac{-x_{n}}{\left\|x_{n}\right\|} d t\right] \\
& \geq \int_{I_{+}} \liminf _{n \rightarrow \infty} \frac{F\left(t, x_{n}\right)}{x_{n}} \frac{x_{n}}{\left\|x_{n}\right\|} d t-\int_{I_{-}} \limsup _{n \rightarrow \infty} \frac{F\left(t, x_{n}\right)}{x_{n}} \frac{-x_{n}}{\left\|x_{n}\right\|} d t .
\end{aligned}
$$


Thus, by a simple computation, we have

$$
\begin{aligned}
& \liminf _{n \rightarrow \infty} \int_{0}^{2 \pi} \frac{F\left(t, x_{n}\right)}{\left\|x_{n}\right\|} d t \\
& \geq \frac{1}{\sqrt{\left(m^{2}+1\right) \pi}} \int_{0}^{2 \pi}\left[F_{+}(t) \sin ^{+}(m t+\theta)-F_{-}(t) \sin ^{-}(m t+\theta)\right] d t .
\end{aligned}
$$

Hence, it follows from (3.13) and (3.14) that

$$
\begin{aligned}
& \sum_{j=1}^{p} J_{j}^{+}\left(t_{j}\right) \sin ^{+}\left(m t_{j}+\theta\right)-\sum_{j=1}^{p} J_{j}^{-}\left(t_{j}\right) \sin ^{-}\left(m t_{j}+\theta\right)+\int_{0}^{2 \pi} e(t) \sin (m+\theta) d t \\
& \geq \int_{0}^{2 \pi}\left[F_{+}(t) \sin ^{+}(m t+\theta)-F_{-}(t) \sin ^{-}(m t+\theta)\right] d t .
\end{aligned}
$$

This contradicts $\left(\mathrm{H}_{3}\right)$. It implies that the sequence $\left(x_{n}\right)$ is bounded. Thus, there exists $x_{0} \in$ $H$ such that $x_{n} \rightarrow x_{0}$ weakly in $H$. Due to the compact embedding $H \hookrightarrow L^{2}(0,2 \pi)$ and $H \hookrightarrow C(0,2 \pi)$, going to a subsequence,

$$
x_{n} \rightarrow x_{0} \quad \text { in } L^{2}(0,2 \pi), \quad x_{n}>x_{0} \text { in } C(0,2 \pi) .
$$

From (3.3), we obtain

$$
\lim _{\substack{n \rightarrow \infty \\ i \rightarrow \infty}}\left(\int_{0}^{2 \pi}\left(\left(x_{n}^{\prime}-x_{i}^{\prime}\right) z_{k}^{\prime}-\eta^{2}\left(x_{n}-x_{i}\right) z_{k}\right) d t-\int_{0}^{2 \pi}\left(f\left(t, x_{n}\right)-f\left(t, x_{i}\right)\right) z_{k} d t\right.
$$

$$
\left.+\sum_{j=1}^{p}\left(I_{j}\left(t_{j}, x_{n}\left(t_{j}\right)\right)-I_{j}\left(t_{j}, x_{i}\left(t_{j}\right)\right)\right) z_{k}\left(t_{j}\right)\right)=0 .
$$

Replacing $z_{k}$ by $x_{n}-x_{i}$ in the above equality, we get

$$
\begin{aligned}
& \lim _{\substack{n \rightarrow \infty \\
i \rightarrow \infty}}\left(\int_{0}^{2 \pi}\left(\left(x_{n}^{\prime}-x_{i}^{\prime}\right)^{2}-m^{2}\left(x_{n}-x_{i}\right)^{2}\right) d t-\int_{0}^{2 \pi}\left(f\left(t, x_{n}\right)-f\left(t, x_{i}\right)\right)\left(x_{n}-x_{i}\right) d t\right. \\
& \left.\quad+\sum_{j=1}^{p}\left(I_{j}\left(t_{j}, x_{n}\left(t_{j}\right)\right)-I_{j}\left(t_{j}, x_{i}\left(t_{j}\right)\right)\right)\left(x_{n}\left(t_{j}\right)-x_{i}\left(t_{j}\right)\right)\right)=0 .
\end{aligned}
$$

By $\left(\mathrm{H}_{1}\right)$ and $\left(\mathrm{H}_{2}\right)$, we have

$$
\lim _{\substack{n \rightarrow \infty \\ i \rightarrow \infty}} \int_{0}^{2 \pi}\left(f\left(t, x_{n}\right)-f\left(t, x_{i}\right)\right)\left(x_{n}-x_{i}\right) d t=0
$$

and

$$
\lim _{\substack{n \rightarrow \infty \\ i \rightarrow \infty}} \sum_{j=1}^{p}\left(I_{j}\left(t_{j}, x_{n}\left(t_{j}\right)\right)-I_{j}\left(t_{j}, x_{i}\left(t_{j}\right)\right)\right)\left(x_{n}\left(t_{j}\right)-x_{i}\left(t_{j}\right)\right)=0 .
$$


Thus, it follows from (3.15), (3.16), and (3.17) that

$$
\lim _{\substack{n \rightarrow \infty \\ i \rightarrow \infty}} \int_{0}^{2 \pi}\left[\left(x_{n}^{\prime}-x_{i}^{\prime}\right)^{2}-m^{2}\left(x_{n}-x_{i}\right)^{2}\right] d t=0 .
$$

Therefore,

$$
\lim _{\substack{n \rightarrow \infty \\ i \rightarrow \infty}}\left\|x_{n}-x_{i}\right\|^{2}=0
$$

which implies $x_{n} \rightarrow x_{0}$ in $H$. It shows that $\varphi$ satisfies (PS).

Now, we can give the proof of Theorem 1.1.

\section{Proof of Theorem 1.1 Denote}

$H^{-}=\mathbb{R} \oplus \operatorname{span}\{\sin t, \cos t, \sin 2 t, \cos 2 t, \ldots, \sin m t, \cos h t\}$

and

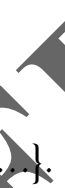

We first prove that

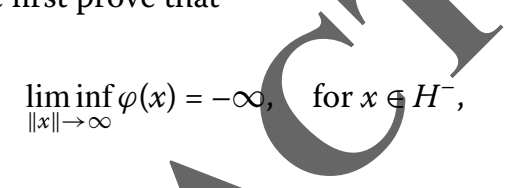

by contradiction Assume that there exists a sequence $\left(x_{n}\right) \subset H^{-}$such that $\left\|x_{n}\right\| \rightarrow \infty$ (as $n \rightarrow \infty$ ) and there exists a constant $c_{-}$satisfying

$$
\liminf \varphi\left(x_{n}\right) \geq c_{-} \text {. }
$$

By $\left(\mathrm{H}_{1}\right)$,we have

$$
\lim _{n \rightarrow \infty} \int_{0}^{2 \pi} \frac{F\left(t, x_{n}\right)-e(t) x_{n}}{\left\|x_{n}\right\|^{2}} d t=0 .
$$

By $\left(\mathrm{H}_{2}\right)$, we get

$$
\lim _{n \rightarrow \infty} \sum_{j=1}^{p} \frac{J_{j}\left(t_{j}, x_{n}\left(t_{j}\right)\right)}{\left\|x_{n}\right\|^{2}}=0 .
$$

From (3.19) and the definition of $\varphi$, we obtain

$$
\begin{aligned}
& \liminf _{n \rightarrow \infty}\left[\frac{1}{2} \int_{0}^{2 \pi} \frac{x_{n}^{\prime 2}-m^{2} x_{n}^{2}}{\left\|x_{n}\right\|^{2}} d t-\int_{0}^{2 \pi} \frac{F\left(t, x_{n}\right)-e(t) x_{n}}{\left\|x_{n}\right\|^{2}} d t+\sum_{j=1}^{p} \frac{J_{j}\left(t_{j}, x_{n}\left(t_{j}\right)\right)}{\left\|x_{n}\right\|^{2}}\right] \\
& \quad \geq 0 .
\end{aligned}
$$


For $x \in H^{-}$, we have

$$
\int_{0}^{2 \pi}\left(x^{\prime 2}-m^{2} x^{2}\right) d t=\|x\|^{2}-\left(m^{2}+1\right)\|x\|_{2}^{2} \leq 0 .
$$

The equality in (3.23) holds only for

$$
x=\frac{1}{\sqrt{\left(m^{2}+1\right) \pi}} \sin (m t+\theta), \quad \theta \in \mathbb{R} .
$$

Set $y_{n}=\frac{x_{n}}{\left\|x_{n}\right\|}$. Since $\operatorname{dim} H^{-}<\infty$, going to a subsequence, there exists $y_{0} \in H^{-}$such that $y_{n} \rightarrow y_{0}$ in $H$ and $y_{n} \rightarrow y_{0}$ in $L^{2}(0,2 \pi)$. Then (3.20), (3.21), (3.22), and (3.23) implythat

$$
y_{0}=\frac{1}{\sqrt{\left(m^{2}+1\right) \pi}} \sin (m t+\theta), \quad \theta \in \mathbb{R}
$$

By (3.19), we have, for $n$ large enough,

$$
\frac{1}{2} \int_{0}^{2 \pi} \frac{x_{n}^{\prime 2}-m^{2} x_{n}^{2}}{\left\|x_{n}\right\|} d t-\int_{0}^{2 \pi} \frac{F\left(t, x_{n}\right)-e(t) x_{n}}{\left\|x_{n}\right\|} d t+\sum_{j=1}^{p} \frac{\left(t_{j}, x_{n}\left(t_{j}\right)\right)}{\left\|x_{n}\right\|} \geq \frac{c_{-}}{\left\|x_{n}\right\|} .
$$

It follows from $x_{n} \in H^{-}$that

$$
\int_{0}^{2 \pi} \frac{x_{n}^{\prime 2}-m^{2} x_{n}^{2}}{\left\|x_{n}\right\|} \leq 0
$$

From (3.24) and (3.25), we get, for $y$ large enough,

Thus,

$$
\frac{c_{-}}{\left\|x_{n}\right\|} \leq-\int_{0}^{2 \pi} \frac{F\left(t, x_{n}\right)-e(t) x_{n}}{\left\|x_{n}\right\|} d t+\sum_{j=1}^{p} \frac{J_{j}\left(t_{j}, x_{n}\left(t_{j}\right)\right)}{\left\|x_{n}\right\|} .
$$

$$
\liminf _{n \rightarrow \infty} \int_{0}^{2 \pi}\left(\frac{F\left(t, x_{n}\right)}{x_{n}}-e(t)\right) \frac{x_{n}}{\left\|x_{n}\right\|} d t \leq \liminf _{n \rightarrow \infty} \sum_{j=1}^{p} \frac{J_{j}\left(t_{j}, x_{n}\left(t_{j}\right)\right)}{\left\|x_{n}\right\|}
$$

Using an argument similar to the proof of Lemma 3.1, we get

$$
\begin{aligned}
& \sum_{j=1}^{p} J_{j}^{+}\left(t_{j}\right) \sin ^{+}\left(m t_{j}+\theta\right)-\sum_{j=1}^{p} J_{j}^{-}\left(t_{j}\right) \sin ^{-}\left(m t_{j}+\theta\right)+\int_{0}^{2 \pi} e(t) \sin (m t+\theta) d t \\
& \quad \geq \int_{0}^{2 \pi}\left(F_{+}(t) \sin ^{+}(m t+\theta)-F_{-}(t) \sin ^{-}(m t+\theta)\right) d t,
\end{aligned}
$$

which is a contradiction to $\left(\mathrm{H}_{3}\right)$.

Then (3.18) holds.

Next, we prove that

$$
\lim _{\|x\| \rightarrow \infty} \varphi(x)=\infty, \quad \text { for all } x \in H^{+}
$$

and $\varphi$ is bounded on bounded sets. 
Because of the compact embedding of $H \hookrightarrow C(0,2 \pi)$ and $H \hookrightarrow L^{2}(0,2 \pi)$, there exists constants $m_{1}, m_{2}$ such that

$$
\|x\|_{\infty} \leq m_{1}\|x\|, \quad\|x\|_{2} \leq m_{2}\|x\| .
$$

Then by $\left(\mathrm{H}_{1}\right)$ and $\left(\mathrm{H}_{2}\right)$, one has

$$
\begin{aligned}
|\varphi(x)|= & \mid \frac{1}{2} \int_{0}^{2 \pi} x^{\prime 2} d t-\frac{m^{2}}{2} \int_{0}^{2 \pi} x^{2} d t-\int_{0}^{2 \pi}[F(t, x)-e(t) x] d t \\
& +\sum_{j=1}^{p} J_{j}\left(t_{j}, x\left(t_{j}\right)\right) \mid \\
\leq & \frac{1}{2}\|x\|^{2}+\frac{m^{2}}{2} m_{2}^{2}\|x\|^{2}+\int_{0}^{2 \pi}(|p(t)||x|+|e(t)||x|) d t \\
& +\sum_{j=1}^{p} c_{j}\left|x\left(t_{j}\right)\right| \\
\leq & \frac{1+m^{2} m_{2}^{2}}{2}\|x\|^{2}+m_{1}\left(\|p\|_{1}+\# e \|_{1}\right)\|x\|+\sum_{j=1}^{p} c_{j} m_{1}\|x\| .
\end{aligned}
$$

Hence, $\varphi$ is bounded on bounded sets of $H$.

Since $x \in H^{+}$, we have

$$
\|x\|^{2} \geq\left((m+1)^{2}+1\right)\|x\|_{2}^{2} .
$$

Thus, from (3.26) and (3.27), we obtain

$$
\begin{aligned}
& \geq \frac{2 m+1}{2\left((m+1)^{2}+1\right)}\|x\|^{2}-m_{1}\left(\|p\|_{1}+\|e\|_{1}+\sum_{j=1}^{p} c_{j}\right)\|x\|, \\
& \text { which implies } \\
& \lim _{\|x\| \rightarrow \infty} \varphi(x)=\infty, \quad \text { for all } x \in H^{+} .
\end{aligned}
$$

Up to now, the conditions (a) and (b) of Theorem 2.2 are satisfied. According to Lemma 3.1, (c) is also satisfied. Hence, by Theorem 2.2, (1.1) has at least one solution. This completes the proof.

\section{Authors' contributions}

The first author has contributed in obtaining new results and written the whole article. The second author has written the references with BibTeX and formatted the manuscript such that it conforms to the journal style. All authors have also read and approved the final manuscript. 


\section{Author details}

'School of Science, Jiujiang University, Jiujiang, 332005, China. ${ }^{2}$ Department of Scientific Research Management, Jiujiang University, Jiujiang, 332005, China.

\section{Acknowledgements}

The authors would like to express their thanks to the editor of the journal and the referees for their carefully reading of the first draft of the manuscript and making many helpful comments and suggestions which improved the presentation of the paper.

Received: 10 June 2014 Accepted: 5 August 2014 Published: 9 September 2014

\section{References}

1. Lazer, AC: On Schauder's fixed point theorem and forced second order nonlinear oscillations. J. M 421-425 (1968)

2. Lazer, AC, Leach, DE: Bounded perturbations of forced harmonic oscillators at resonance. Ann. Mat. Pura Appl. 82(4), 49-68 (1969)

3. Landesman, EM, Lazer, AC: Nonlinear perturbations of linear elliptic boundary value problems at resonanCe. J. Math. Mech. 19, 60-623 (1970)

4. Lakshmikantham, V, Bainov, DD, Simeonov, PS: Theory of Impulsive Differential Equations. World Scyientific, Singapore (1989)

5. Bogun, I: Existence of weak solutions for impulsive $p$-Laplacian problem with superlinearimpulses. Nonlinear Anal., Real World Appl. 13(6), 2701-2707 (2012)

6. Ding, W, Qian, D: Periodic solutions for sublinear systems via variational approach. Nonlinear Anal., Real World Appl. 11(4), 2603-2609 (2010)

7. Zhang, Z, Yuan, R: An application of variational methods to Dirichlet boundary value problem with impulses. Nonlinear Anal., Real World Appl. 11(1), 155-162 (2010)

8. Nieto, JJ, O'Regan, D: Variational approach to impulsive differential equations. Nonlinear Anal., Real World Appl. 10(2), 680-690 (2009)

9. Zhang, H, Li, Z: Variational approach to impulsive differential equations with periodic boundary conditions. Nonlinear Anal., Real World Appl. 11(1), 67-78 (2010)

10. Zhang, X, Meng, Q: Nontrivial periodic solutions fol delay differential systems via Morse theory. Nonlinear Anal. 74(5), 1960-1968 (2011)

11. Tomiczek, P: The Duffing equation with the potentiak Landesman-Lazer condition. Nonlinear Anal. 70(2), 735-740 (2009)

12. Mawhin, J, Willem, M: Critical Point Theory and Hamiltonian Systems. Springer, Berlin (1989)

13. Rabinowitz, PH: Minmax Methods in Critical Poikt Theory with Applications to Differential Equations. CBMS Reg. Conf. Ser. in Math., vol. 65. Am. Math. Soc., Providence (1986)

14. Fučík, S: Solvability of Nonlinear Equations and Boundary Value Problems. D. Reidel Publ. Company, Holland (1980)
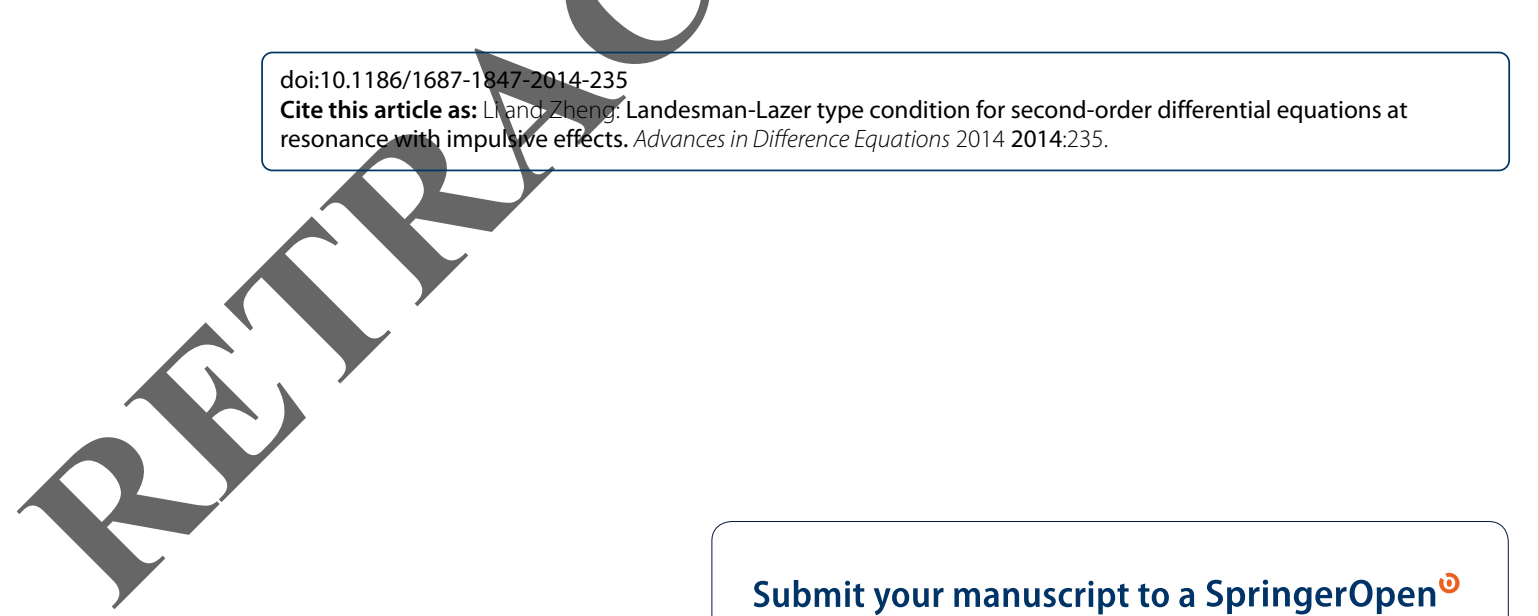

\section{Submit your manuscript to a SpringerOpen ${ }^{\circ}$ journal and benefit from:}

- Convenient online submission

Rigorous peer review

- Immediate publication on acceptance

Open access: articles freely available online

- High visibility within the field

- Retaining the copyright to your article 